\title{
NIVEL DE ESTRES DE LAS MADRES CON RECIEN NACIDOS HOSPITALIZADOS EN LA UNIDAD DE CUIDADOS INTENSIVOS NEONATAL, HOSPITAL GUILLERMO GRANT BENAVENTE DE CONCEPCION, CONCEPCION, 1999
}

\author{
MOTHER'S STRESS LEVEL OF NEWBORN IN NEONATAL INTENSIVE CARE \\ UNIT AT THE GUILLERMO GRANT BENAVENTE HOSPITAL, CONCEPCION, \\ CHILE, 1999
}

VIVIANE EUGENIA JOFRÉ ARAVENA , ELENA HENRÍQUEZ FIERRO

\begin{abstract}
RESUMEN
Estudio descriptivo correlacional cuyo propósito fue determinar el nivel de estrés de las madres de los recién nacidos hospitalizados en la Unidad de Cuidados Intensivos Neonatal del Hospital Guillermo Grant Benavente de Concepción, en Concepción, Chile, 1999. La muestra accidental estuvo constituida por 35 madres de los recién nacidos hospitalizados. Para dar respuesta al propósito de la investigación, se aplicaron los instrumentos: Escala de Estrés Maternal, que mide el nivel de estrés y las dimensiones de él; y el cuestionario de antecedentes biosociodemográficos de las madres y características de los recién nacidos. Del total de las variables planteadas, resultaron relacionadas con el nivel de estrés de las madres: el ambiente de la unidad; el aspecto y comportamiento del recién nacido y el rol de la madre. Respecto a las variables independientes biosociodemográficas resultaron no estar relacionadas con el estrés total de las madres, al igual que las variables relacionadas con las características del recién nacido. Con los resultados obtenidos es conveniente replantearse la participación de la madre en la atención de los recién nacidos prematuros y enfermos, ya que a la luz de los hallazgos se visualiza esta necesidad. Por otra parte, el profesional de enfermería debe estar sensible a reconocer las características particulares e individuales de cada madre para la intervención correspondiente, según lo amerite el caso.
\end{abstract}

Palabras claves: Estrés, Madres, Recién nacido, Unidad de Cuidado Intensivo Neonatal.

\begin{abstract}
The aim of this descriptive correlational study was to determine the Stress Level in mothers of newborns from the Neonatal Intensive Care Unit at the Guillermo Grant Benavente Hospital, Concepción, Chile, 1999.

Thirty five mothers of hospitalized newborns were considered for an accidental sample.

The following instruments were applied to accomplish the research purpose: The Maternal Stress Scale that measures the stress level, and its dimension; The Biosociodemographic's Records of mother and Newborns Characteristics Questionnaire.

From the variables proposed, the unit's environment, the newborn's aspect and behaviour, the mother's role, turned out to be related to the mother's stress level.

As for the biosociodemographic variables, they were not related to the mother's total stress as well as the newborn's characteristics.

Considering the results as obtained, it is convenient to think over about the mother's participation in the premature and ill newborn care, since according to the findings, the need is recognized. On the other hand, the nurse as professional, must be sensitive to recognize the particular and unique characteristics of every mother for the intervention as the case might deserve it.
\end{abstract}

Keywords: Stress, Mothers, Newborn, Neonatal Intensive Care Unit.

${ }^{1}$ Profesor Asociado, Enfermera, Enfermera Especialista en Enfermería Pediátrica. Magister en Enfermería con mención en Salud Comunitaria Dpto. de Enfermería, Universidad de Concepción, Concepción Chile.

${ }^{2}$ Profesor Titular, Enfermera, Enfermera Magister en Enfermería con mención en Salud Comunitaria, Dpto. de Enfermería, Universidad de Concepción, Chile. 


\section{INTRODUCCION}

El nacimiento de un niño o niña inevitablemente trae una serie de cambios que alteran la rutina cotidiana y que producen estrés en los miembros de una familia, siendo éste considerado generalmente un acontecimiento positivo. Estos cambios pueden ser percibidos como negativos y provocar una fuerte tensión al interior del núcleo familiar.

Cuando el nacimiento ocurre antes del tiempo esperado o nace un recién nacido (RN) enfermo, puede ser aún más estresante para una familia, por muchas razones. Estas incluyen el percibir la sensación de fracaso de tener un niño enfermo, el temor a la muerte del niño, la alteración del apego niño-padres, la interrupción en la rutina familiar, el temor con respecto a su cuidado y protección y resignarse a aceptar que otras personas cuiden de él en este período.

Con la mayor instrumentalización y con la implementación de las Unidades de Cuidados Intensivos Neonatal (UCIN) se abren mayores expectativas de vida a aquellos RN de escaso peso de nacimiento. Junto con la preocupación de mantener con vida a este pequeño ser, el profesional de enfermería debe, desde el momento que el RN se hospitaliza, intentar incorporar al niño que nace con problemas a su familia, estimulando la visita de sus padres, promoviendo la mantención de la lactancia materna, tratando de incorporar a los padres al cuidado de su hijo informando su evolución y estado de salud, para aminorar la angustia y desesperanza, así como fomentar los lazos afectivos que se mantendrán durante toda la vida.

La atención crítica en las UCIN es altamente estresante para los padres, especialmente para las madres, por lo cual se hace necesario identificar el nivel específico de estrés y las fuentes que producen esta tensión para desarrollar intervenciones de enfermería efectivas para ayudarlos a enfrentar favorablemente esta situación.
Este estudio se basa en la Teoría del Estrés de Lazarus (1986) que plantea "estrés psicológico en una relación particular entre el individuo y el entorno, que es evaluado por éste como agravando o desbordando sus recursos y que pone en peligro su bienestar"1.

La definición de Lazarus considera como fundamental la relación individuo-entorno.

En el estrés no siempre es suficiente la sola situación estresante, sino que procesos de afrontamiento inadecuados en el individuo pueden llevar a un resultado no esperado o no deseado (respuesta disfuncional).

En el contexto de esta teoría tenemos un proceso evaluativo que determina, a la luz de los factores de la persona y de la situación, por qué y hasta qué punto una relación determinada o una serie de relaciones entre el individuo y el entorno es estresante.

Entre los antecedentes empíricos más relevantes cabe mencionar Shields-Poe, D.; Pinelli, J. (1997) quienes realizaron un estudio descriptivo para identificar las fuentes de estrés parental en dos tipos de UCIN; ellos encontraron que la variable más poderosa relacionada con el estrés era la forma en que los padres percibían la severidad de la enfermedad de su hijo, además cuándo y dónde los padres vieron por primera vez al niño.

Meyer, E.; García et col (1995) realizaron un estudio para identificar las características del RN prematuro que ingresa a las UCIN y las de sus madres que producen exceso de estrés en ellas. Fueron identificadas como fuentes de estrés, las características del niño tales como: peso, edad gestacional y apoyo respiratorio, referido esto a la ventilación mecánica.

Miles, Ms.; Funk, Sg.; Kasper Ma. (1991) encontraron que las alteraciones del rol parental causada por la enfermedad del RN generó un alto grado de estrés en los padres. La segunda causa la constituyó la apariencia y comportamiento del RN hospitalizado en una UCIN.

${ }^{1}$ Lazarus, R. y Folkman, S. Estrés $\underline{y}$ y prococeso $\underline{s}$ cognitivos. Martínez Roca, 1986, pp. 25-117. 


\section{PROPOSITOS Y OBJETIVOS}

\section{Propósito}

Determinar el nivel de estrés de las madres de los RN hospitalizados en la UCIN del Hospital Guillermo Grant Benavente de Concepción (HGGBC) y los factores que se relacionan con él.

\section{Objetivos}

- Determinar el nivel de estrés de las madres de los RN hospitalizados en la UCIN del HGGBC.

- Identificar las dimensiones que influyen en el nivel de estrés de las madres con RN hospitalizados en la UCIN.

- Identificar los factores biosociodemográficos que se relacionan con el nivel de estrés de las madres con RN hospitalizados en la UCIN del HGGBC.

- Identificar las características del RN que se relacionan con el nivel de estrés de las madres.

\section{MATERIAL Y METODO}

Estudio descriptivo correlacional, realizado entre los meses de junio a diciembre de 1999 en la UCIN del HGGBC, que buscó identificar el nivel de estrés y los factores que se relacionan con él, en una población de madres con RN hospitalizados en la UCIN del HGGBC.

\section{INSTRUMENTOS RECOLECTORES DE DATOS}

Para obtener los datos necesarios se utilizaron los siguientes instrumentos:

1. Escala de Estrés Maternal (EEM). Esta escala fue elaborada por la investigadora, sobre la base de una escala de las autoras C. Margaret, S. Miles, RN Ph D. realizada en USA en 1987, denominada "Escala de Estrés Parental: Unidad de Cuidados Intensivos Neonatal", validado para población norteamericana, motivo por el cual fue modificada para las características de nuestra población y de acuerdo a la realidad de nuestros servicios hospitalarios y también basada en la opinión de profesionales de enfermería docentes y clínicos con vasta experiencia en el área de los cuidados intensivos pediátricos y neonatales. Posterior a la aplicación de la prueba piloto, se modificaron algunos ítemes de acuerdo a los resultados de los coeficientes de Cronbach, previa consulta con dos profesionales psicólogos con experiencia en el trabajo con familias de pacientes hospitalizados. Este instrumento es respondido con escala Likert de 4 puntos, que va desde 1 (sin estrés) a 4 (estrés excesivo). Consta de cuatro subescalas que miden el nivel de estrés de las madres frente:

a) Ambiente de la UCIN.

b) Aspecto del RN.

c) Rol de la madre, y

d) Comunicación con el personal

Finalmente, la escala posee una pregunta referente a cuánto ha sido en general el estrés generado por la situación de tener un RN hospitalizado en la UCIN, usando la misma escala.

2. Cuestionario de antecedentes biosociodemográficos. Este cuestionario fue confeccionado por la investigadora del estudio y recopiló datos necesarios para sustentar esta investigación. Consta de dos partes: la primera, para recopilar los antecedentes biosociodemográficos de las madres; la segunda parte consta de ítemes para determinar las características de los RN hospitalizados en la UCIN.

\section{POBLACION EN ESTUDIO}

Universo. Todas las madres de los RN hospitalizados en la UCIN del HGGB de Concepción entre el $1^{\circ}$ de junio de 1999 y el 31 de 
diciembre de 1999. El universo real quedó constituido por 65 madres.

Muestra. Es de tipo accidental y correspondió al 53,8\% del universo real, equivalente a 35 casos.

\section{PROCESAMIENTO DE LOS DATOS}

El instrumento recolector de datos fue codificado y procesado computacionalmente, utilizando el programa estadístico SPSS para las ciencias sociales, en sus diferentes capacidades de procesamiento estadístico.

Para el análisis estadístico de este estudio y para responder a las interrogantes de la investigación, considerando que se realizó una medición multivariada del estrés sobre la base de cuatro subescalas, se analizó el grado de correlación que existe entre ellas, usando análisis de correlación.

Se comparó el grado de estrés de las madres, medido a través de la última pregunta de la EMM, con los puntajes obtenidos en las distintas subescalas, usando tablas de contingencia.

Se usó chi cuadrado de Pearson para ver si existe grado de asociación y la fuerza de ésta con el coeficiente $\mathrm{V}$ de Cramer, y en las tablas de contingencia de las variables biosociodemográficas en relación con cada una de las subescalas de estrés para medir el grado de asociación entre ellas.

Para la confiabilidad del instrumento EMM se utilizó el coeficiente de correlación Alpha de Cronbach, que resultó de 0,87 , que muestra que existe alta confiabilidad entre los ítems.

\section{RESULTADOS}

$\mathrm{Al}$ analizar las correlaciones de las cuatro subescalas, que constituyen las dimensiones de la variable dependiente, resultaron significativa al nivel 0.01 el aspecto del RN relacionado con el ambiente de la UCIN; rol materno en relación con el aspecto del $\mathrm{RN}$; comunicación con el personal de la UCIN relacionado con el aspecto del $\mathrm{RN}$ y rol materno en relación con el ambiente de la UCIN; y al nivel 0.05 resultó significativa la correlación entre comunicación con el personal relacionado con el ambiente de la UCIN. No existe correlación significativa entre las variables comunicación con el personal y el rol materno.

Respecto a las variables biosociodemográficas de las madres, resultó una relación estadísticamente significativa entre el nivel educacional y la dimensión del estrés total, rol de la madre; y la situación marital con la misma dimensión, el resto de las variables biosociodemográficas y las características del RN hospitalizado en la UCIN resultaron no estar relacionadas con el estrés de las madres y sus dimensiones.

\section{DISCUSION Y COMENTARIOS}

Las madres cuyos $\mathrm{RN}$ requieren hospitalización en la UCIN, experimentan diversos niveles de estrés. El personal que trabaja en estas unidades, y principalmente el profesional de enfermería, necesita estar consciente de este estrés que experimentan las madres que pueden influenciar su capacidad para oír explicaciones, tomar decisiones e involucrarse con su pequeño niño.

En este estudio se encontró que todas las correlaciones de las dimensiones de estrés resultaron significativas, salvo entre las variables comunicación con el personal y rol de las madres; estos hallazgos son coincidentes con estudios previos entre los cuales se puede mencionar el de Paludetto y colaboradores (1981), quienes encontraron que el ambiente físico y psicosocial de la UCIN alteraba a los padres.

En otro estudio realizado por Miles y col (1991) destacan que la mayoría de los padres presentan niveles moderados de estrés, concluyendo que el ambiente que rodea al RN hospitalizado, tanto físico como social, se relaciona significativamente con la ansiedad de los padres. 
Respecto a los niveles de estrés de las madres, $51,4 \%$ presentó niveles estresantes y $48,6 \%$ niveles muy estresantes, lo que avala el hecho que aunque el nacimiento de un nuevo ser es un acontecimiento esperado, genera un cierto nivel de estrés en las madres, y más aún cuando el RN nace antes de lo esperado, tiene que estar hospitalizado y no puede permanecer al lado de su familia. Estos resultados son coincidentes con un estudio preliminar realizado por Jofré y Henríquez en 1998, en que se solicitó a los padres que expresaran sus sentimientos al ser separados de sus RN. Ellos manifestaron, en un $100 \%$, sentimientos que revelan cierto nivel de estrés; mayoritariamente $(33,3 \%)$ manifestaron miedo, 29,2\% angustia y desesperación y $8,3 \%$ se sintió inútil.

En la dimensión del estrés que dice relación con el ambiente de la UCIN, esta variable resultó influyente en el nivel de estrés total de las madres, ya que se relaciona en forma significativa; estos resultados son concordantes con los encontrados por Shields y colaboradores (1997), quienes describieron como variable poderosa relacionada con el estrés el lugar donde los padres vieron a su hijo.

El aspecto del RN también resultó un factor relacionado en el estrés de las madres, obteniendo una relación altamente significativa con la variable estrés total, lo que concuerda con los datos obtenidos por Miles y colaboradores (1991). Ellos describieron como segunda causa productora de un alto grado de estrés en los padres, la apariencia y comportamiento del RN hospitalizado en UCIN.

La dimensión rol materno también resultó relacionado con el nivel de estrés total con una significación estadística de 5\%. Al igual que en el estudio de Miles y col. (1991), en el cual uno de los aspectos más estresantes lo constituye la alteración del rol de los padres, sintiéndose inútiles de no poder ayudar a su hijo.

La comunicación con el personal resultó ser una dimensión no relacionada estadísticamente con el estrés total, lo que sería similar a lo pesquisado por la investigadora en el estudio "Sentir de los padres con RN hospitalizado en UCIN" (1998), en el cual los padres manifestaron, mayoritariamente, sentimientos positivos hacia la relación con el personal.

La edad de las madres resultó no estar relacionada en forma estadísticamente significativa, a pesar de que los datos mostraban que al parecer, a mayor edad, existían mayores niveles de estrés. Lo cual es contrario a los hallazgos del estudio de Meyer y col. (1995), en el cual describieron que la menor edad de la madre es predictiva de estrés psicológico general en la situación de tener un hijo hospitalizado.

Respecto al nivel educacional y la dimensión del estrés, rol de la madre, estas variables resultaron estar estadísticamente relacionadas.

Con relación al lugar de residencia de las familias de las madres de los RN hospitalizados en la UCIN, se encontró una relación altamente significativa con el nivel de estrés.

Las otras características biosociodemográficas de las madres de los RN hospitalizados en la UCIN tales como: situación marital, religión, tipo de familia, experiencias anteriores, historia obstétrica de la madre no se relacionaron en forma estadísticamente significativa con el nivel de estrés y sus dimensiones, al igual que las variables relacionadas con las características de los RN de las madres del estudio que incluyeron: Edad gestacional, peso del RN, patología y tipo de nacimiento. Esto no concuerda con los estudios de Meyer E., García y col. (1995), donde fueron identificados entre otras, como fuentes de estrés, las características del RN, tales como peso y edad gestacional. Por otro lado, Heuer L. (1993) también había encontrado resultados similares al anterior.

\section{REFERENCIAS BIBLIOGRAFICAS}

Gulseth, K. Factors Influencing the Coping Effort of Mothers of Hospitalized Children en: Nursing Research. 1 (40), enero-febrero 1991, pp. 42-46.

Heuer, L. Parental Stressor in a Pediatrics intensive care unit En: Pediatrics Nursing, USA. 19 (2): 31.128 , marzo-abril 1992. 
Jofré, V. Henríquez, E. Sentir de los padres frente a un RN hospitalizado en UCIN del HGGBC. En Jornadas de Investigación en Enfermería IV, Talca, Chile, 3 de diciembre 1998.

Lazarus, R. Folkman, S. Estrés y procesos cognitivos. Martínez Roca, 1986, pp. 25-117.

Lazarus and Folkman (1984). Stress $\underline{\text { L_ }}_{\text {appraisal }}$ and coping. New York: Springer Publishing Co.

Lynne, A. et al. Maternal Stresses and Depressive Symptoms: Correlates of Behavior Problems in Young Children en: Nursing Research 3 (37), mayojunio 1988, pp. 156-161.

Meyer, Ec. García, Ct. Psychological distress in mother of preterm infants. En: I. Der-Behaivor-Pediatric, USA 16 (6): 412-417, diciembre 1995.

Miles, $\mathrm{M}$ et al. The Neonatal Intensive Care Units environment: Sources of stress for parents. En: AACN-Clinics Issues Critical Care Nursing, USA 2 (2): 346-354, mayo 1991.

Miles, $\mathrm{M}$ et al. Parental Stressor Scale: Neonatal Intensive care unit. En: Nursing Research, USA 42 (3): 148-152, mayo-junio 1993.
Muniagurria. G. Intervenciones Psicosociales en la UCIN “ Cuidando al RN y sus padres" 1998. http:/ / Psicología - Online. com

Padua, J. Técnicas de la Investigación aplicados a las ciencias sociales. $1^{a}$ ed. Santiago, Chile. 1994, Fondo de Cultura Económica.

Paludetto, R. et al. Reactions of sixty parents allowed unrestricted contact with infants in a neonatal intensive care unit. En: Early Human Development, USA, 5 (5): 401-409, 1981.

Polit, D \& Hungler, B. Investigación científica en ciencias de la salud. Edit. Interamericana-McGraw Hill. $4^{a}$ ed., 1994.

Shields et al. Variables asociadas al estrés maternal en unidades de cuidados intensivos neonatales. En: Neonatal Netw, USA, 16 (1): 29-37, febrero 1997.

Walker, L. Stress Process Among Mothers of Infants: Preliminary Model Testing en: Nursing Research 1 (38), enero-febrero 1989.

Youngblut, J et al. Maternal Employment Effects on Family and Preterm Infants at three Months en: Nursing Research 5 ( 40), septiembre-octubre 1991, pp. 272-275. 